\title{
TOWARDS A FRAMEWORK FOR SYSTEMATICALLY ANALYSING COLLABORATIVE ERROR
}

\author{
Angela Miguel and Peter Wright \\ Univeristy of York, Department of Computer Science, Heslington, York, YO10 5DD, UK
}

Abstract: One of the main difficulties in creating a model-based, predictive error analysis method for collaborative work is establishing a useful perspective with which to describe collaborative work and failure systematically. This paper addresses the need for a systematic approach to analysing possible failures of collaborative work in order to create such a method. An iterative approach to the design of the error analysis method has been taken. This paper first discusses the original approach taken to question development for an error analysis of collaborative work, and then focuses on the creation of an improved approach to question development using the results of an evaluation of the original approach as a guide. The new approach involves enhancing the model of collaboration used, breaking collaboration into three aspects (coordination, cooperation and co-construction) and creating a framework to structure a systematic examination of collaboration in terms of cognitive stage

Key words: Collaborative Work; Collaborative Error; Human Error Analysis.

\section{INTRODUCTION}

Although the importance of evaluating safety-critical systems for possible human error is well recognised, few of the many existing error analysis techniques consider collaborative error. Most human error analysis techniques focus on errors that might happen during the interaction between a single individual and the system they are using, despite the fact that most work takes place in groups or teams. Collaborative work is susceptible to errors that emerge as a result of the distributed knowledge that this type of work involves, which places extra demands on participants. Collaborative 
errors may be caused by factors such as a lack of situation awareness or awareness of each other, misunderstandings between participants, conflicts, and failures of co-ordination. An error analysis method that can tackle these issues is required.

It is now widely accepted in the Human Reliability Analysis literature that a model-based approach to analysing human error is the most valid (see for example Hollnagel, 1998). However, a key difficulty in creating a model-based, predictive error analysis method for collaborative work is establishing a systematic description of collaborative work and failure. The systematic error analysis techniques currently available largely ignore collaborative work. Although fields such as Computer Supported Cooperative Work (CSCW) contain many descriptions of collaborative work there are few helpful models and no systematic approaches for identifying possible types of collaborative error and reasons for it. Models for the architecture of groupware do exist (such as Clover (Laurillau and Nigay, 2002)), but these focus on functionality and not error. Therefore they do not provide detailed information about collaboration of the type needed to base an error analysis of collaborative work on.

The process of designing an error analysis method is complex and it is important to consider the user in designing such a method. Thus, learning from HCI approaches to design, an iterative approach to the design of the error analysis method has been taken. The next section of this paper discusses the original approach taken to using a model of collaboration for question development for the CHLOE technique. The problems discovered with this approach through an evaluation are then briefly discussed. The remainder of the paper focuses on how, using the results of the evaluation as a guide, an improved approach to analysing collaborative error (and hence creating error analysis questions) is created for the error analysis technique.

\section{CHLOE}

The CHLOE human error analysis technique (Miguel and Wright, 2003) was developed as a model-based method to analyse collaborative work, and help make redesign suggestions. CHLOE considers issues that are important for collaborative work, which would be missed if using other existing error analysis or evaluation approaches. Analysis techniques not designed for collaborative work miss the combinations of factors that may lead to collaborative error. Following the tradition of model-based error analysis approaches such as THEA (Pocock et al, 2001) and CREAM (Hollnagel, 1998), the CHLOE process consists of several stages. These are: Scenario Description, Goal Decomposition, and Error Analysis. The Error Analysis 
questions are based on a model of collaboration. Finally, Design Issues are considered according to the outcomes of the Error Analysis stage.

\subsection{CHLOE's Model of Collaboration}

The error analysis stage in CHLOE is based on a model developed from a basic framework of collaboration by Dix (1998, p.495) and shows various types of communication involved in collaborative work. Failures in collaboration are framed as being failures in communication and understanding between participants. The model is composed of participants (P), an artefact of work (A), different possible types of communication, and shared understanding which develops through the process of communication. A simple cognitive loop (Norman, 2002) has been added to the basic model to represent the cognition of each participant when interacting with other humans or machines.

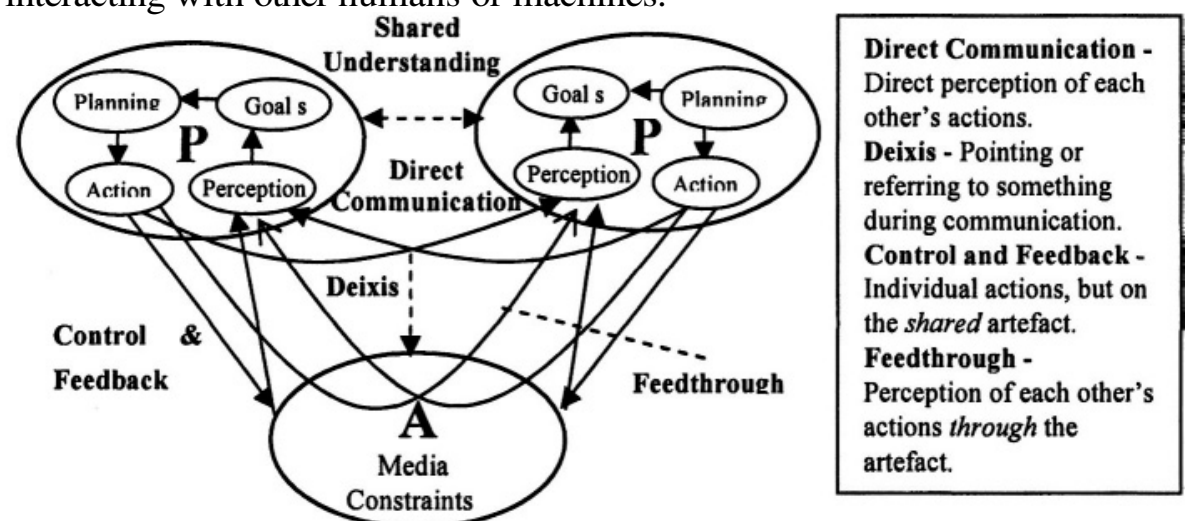

Figure 1.The Model of Communication Used in CHLOE

The arcs in the diagram indicate how the participants and artefacts are linked in each type of communication. All types of communication can help to create and support shared understanding, which helps collaboration to work effectively.

\subsection{Breakdowns in Collaboration}

CHLOE views collaborative failures as stemming from breakdowns in the cognition of the participants involved. Basing the error analysis on failures within a cognitive model of collaboration can help lead to design solutions. This is because it provides a perspective on why the observable failures occur that is useful for helping to consider design requirements. Error analysis questions were developed by applying guidewords describing types of failure to the cognitive stages in the model of collaboration (see 
Table 1). The analysis questions developed from these failures are therefore generally concerned with failures of perception and evaluation, goals, planning, and actions. For example:

- failures of perception/evaluation - e.g. the collaborating participants may not all perceive or interpret the information in the same way, or may not be able to perceive what each other is doing

- failures of goals - e.g. each participant may not know what he/she is supposed to be doing and when, or their goals may conflict

Table 1. Cognitive Stages and Failure Guidewords Used in CHLOE

\begin{tabular}{|l|l|l|l|}
\hline Cognitive Stage & $\begin{array}{l}\text { Failure } \\
\text { Guidewords }\end{array}$ & Cognitive Stage & $\begin{array}{l}\text { Failure } \\
\text { Guidewords }\end{array}$ \\
\hline Perception/Evaluation & $\begin{array}{l}\text { Error } \\
\text { Failure/None }\end{array}$ & $\begin{array}{l}\text { Planning } \\
\text { (Coordination) }\end{array}$ & $\begin{array}{l}\text { Incomplete } \\
\text { Error } \\
\text { Failure/None }\end{array}$ \\
\hline Goals & $\begin{array}{l}\text { Conflict } \\
\text { Error } \\
\text { Failure/None }\end{array}$ & Action & $\begin{array}{l}\text { Error } \\
\text { Failure/None } \\
\text { Conflict } \\
\text { Incomplete }\end{array}$ \\
\hline
\end{tabular}

The guidewords used were selected from the SUSI (Chudleigh and Clare, 1993) modified HAZOP (Kletz, 1999) technique, which was developed to analyse user-system interaction.

The types of failure shown in Table 1 were considered in relation to the types of communication shown in the model to create examples of failure such as, a Failure of Perception in Feedthrough (e.g. one participant cannot see the result of the other's actions) or an Error of Perception in Deixis (e.g. one participant refers to something, but the other participant misunderstands what is being referred to). Forty-eight possible failures were created from the combination of the guidewords, cognitive stages, and types of communication in collaboration according to the model used. Twenty-one questions were then developed around the possible reasons for these failures for each type of communication. Example questions include: (Goals Q5) 'Are participant's goals or sub-goals likely to come into conflict?' and (Planning Q4) 'Is there a shared representation which is consistently visible and understood by all concerned, which can be referred to (e.g. pointing) when sharing information?'

\subsection{Problems with the CHLOE Approach}

As part of the iterative approach taken to the development of the method, a small evaluation of CHLOE was performed using seven participants analysing a short air traffic control scenario. These participants were either $\mathrm{PhD}$ students or Research Associates in the Department of Computer 
Science and had varying amounts of knowledge about Human Factors and Human Error Analysis techniques. They were given an introduction to the CHLOE technique and then provided with written material about both CHLOE and the air traffic control domain. After analysing the air traffic control scenario using CHLOE, the participants completed a questionnaire about the method and its application. The questionnaire was designed to draw out any doubts or dissatisfaction with the method so that improvements can be made. It was split into five sections: modelling, error analysis questions, re-design issues, usability, and effectiveness. Some of the participants were also interviewed later about the answers they had provided in the questionnaire. This was done to clear up ambiguities and collect additional information about their answers.

The evaluation revealed that CHLOE sometimes failed to elicit answers that were specific enough to support detailed failure analysis. It also revealed a lack of consistency between analysts. Vague error analysis questions were a major cause of these problems. These difficulties exist for several reasons. First, the number of error analysis questions is severely constrained by the use of a scenario and task-based approach, which requires questions to be repeated many times over. The amount of effort required for each round of questioning has to be minimized with such an approach, or the overall effort required for analysis would become too great. The questions were kept to a minimum by forcing them to cover a number of issues at once. This increased their tendency to be vague. Second, the few, general failures within the model create too simple a view of collaborative failure to systematically support the development of detailed error analysis questions. Finally, CHLOE uses a model of communication in isolation. It is not clear how the wider collaborative work context affects it.

The results of the evaluation suggest that to be useful, the error analysis questions need to be more precise in the directions they give to users. If more error analysis questions could be supported (by taking a different approach to analysis), questions could be more specific without a loss of coverage of different types of possible errors. To improve the error analysis, a stronger basis for understanding collaborative work (what collaborative error is, what collaborative work involves, and what it requires to be successful) is needed. The model of communication itself needs to be enhanced to provide more detail about what communication involves. This will help the error analysis questions to become more specific. The model of communication also needs to be interpreted within the wider context of collaborative work to allow the systematic construction of the reasons behind failure and the conditions for success. A framework is then needed to structure the systematic identification of possible collaborative failure using this extra information. 
The following two sections explain the approach taken to improve the model of communication and view of collaborative work as a whole. These improvements enable the creation of a framework to identify possible collaborative errors, on which to build a more reliable and effective error analysis method for collaborative work

\section{ENHANCING THE MODEL}

The evaluation results suggested that the model of communication needed to contain more detail about possible types of communication to enable a more thorough analysis of collaborative work. In order to consider how the model needed to be enhanced, a number of types of collaborative work were examined (for example, work in a neo-natal intensive care unit). The basic Dix model (1998) was used as a basis to structure a categorisation of such collaborative work. It was established that changes to the model were needed in the following ways.

Firstly, directly communicating face-to-face is not considered separately from communicating through a video conferencing system or using email. These are all examples of what is labelled Direct Communication. There are however important differences in these forms of communication that will affect collaboration. These differences (for example temporal and spatial differences) may become important when considering possible errors in collaboration and how the design of the system helps to prevent these. The mode of communication may be important in an error analysis. Mediated communication should therefore be recognised as separate from direct faceto-face communication. The model of communication can easily be altered to do this (see the extra line labelled M linking the participants in Figure 2). The line indicating feedthrough has also been altered to indicate that feedthrough occurs as a result of the interactions of one person with the shared artefact.

The second limitation of the model is that it shows only two participants for the sake of simplicity. Direct communication may be one-to-one as the model depicts, but consideration needs to be given to how communication may be altered if there are more than two participants involved. It is important to consider whether communication is to one, some, or all of the group. It is also necessary to examine whether communication is direct and specific, or general (such as verbalisation or talking out loud). These issues are interesting because they relate to observation and overhearing issues, which are recognised as important for collaborative work because they affect awareness (for example Clark (1996) and Segal (1995)). Segal refers to the unintentional communication that results from deliberate communication 
and interaction between participants and artefacts in collaborative work as 'consequential communication'. It is a crucial ingredient for helping collaborative work to function effectively. It is therefore important to establish how these issues relate to the model of communication being used. The level of analysis that the model uses, the elements involved and the terms in which collaboration is described are particularly well suited to the consideration of these issues.

Using the model, possible patterns of observation can be considered in relation to the types of communication shown. This covers both observation of the actions themselves and the results of actions. This information is generally important for awareness concerns, but also more specifically for issues such as checking, reacting to one another's actions, and supporting each other. The ability to observe control and feedback or observe actions through feedthrough may be used deliberately as a means of non-verbal directed communication, or it may simply be a useful side-product of actions that help awareness of others' actions. Observation and overhearing can also be taken a step further to consider the ability for one, some, or all of the group to overhear or see interactions between other participants.

It is also important to consider deixis in relation to overhearing and observation issues. It can be either purely verbal or involve pointing at something, and this may be influenced by the type of communication that it is part of. Deixis may involve an artefact that is visible for all participants communicating, for only one or some of those participating, or it may not be present at all. For this last example, the artefact that is being referred to is not necessarily a physical artefact, but rather something such as procedures or rules (what activity theorists would refer to as psychological artefacts (Nardi, 1996)).

Considering issues such as observation and overhearing in relation to the model of communication allows it to deal better with both intentional and unintentional communication. It can therefore be used more effectively to account for both verbal and non-verbal communication between participants.

Finally, more detail is required about shared understanding. Further enhancement can be made to the model by specifying in more detail what participants may need to develop shared understanding about (for example about each other, or about the work situation). A useful way to structure a more detailed examination of shared understanding is to break it down into past, present and future issues. Participants in collaborative work may require to interpret their interactions with one another in terms of what has already happened (the past), what the current situation is (the present) and/or what they believe the future will be or what they want to it be (team goals) (the future). This refinement of the model of communication enables a more 
specific and systematic identification of where shared understanding may fail (Figure 2).

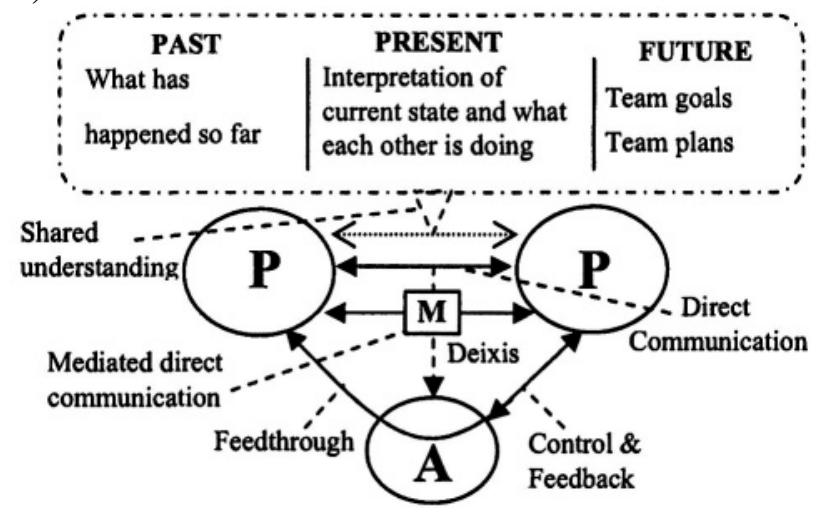

Figure 2. Possible New Version of the Dix Model of Collaboration (the cognitive stages for $\mathrm{P}$ are omitted for the sake of simplicity)

There is more to collaboration than just communication and interaction. Having created a more detailed view of communication in collaboration, to understand what is required of these communications it is necessary to take a wider view of what shapes them.

\section{A BREAKDOWN OF COLLABORATION}

The model and approach used in CHLOE to create possible failure types resulted in an error analysis that does not effectively capture what is necessary for collaboration to work successfully and what a failure of collaboration may be. This is because the model used examined the communication and interaction involved in collaborative work in isolation. It included no representation of the larger activity of which communication and interaction are parts, which makes it difficult to interpret the model. The interactions shown in the model may happen for multiple reasons within collaborative work. What is required of these interactions and how they may fail depends on what they are attempting to achieve and in what conditions. It is necessary to begin with a higher-level consideration of what collaboration involves in order to properly examine work at the level of the communications and interactions shown in the model.

Collaborative work has many different types and aspects. Different forms of collaboration may have different requirements to enable success, or causes for error. Splitting work into types using, for example, the time/space matrix (Dix, 1998, p.488) highlights important differences in types of work, but does not provide a suitable structure with which to examine 
collaboration itself. The alternative of dividing collaboration into its component aspects in order to deal with each more specifically was therefore investigated. Through examining work on Distributed Cognition (Hutchins, 1995), Coordination Theory (Malone and Crowston, 1990), Coordination Mechanisms (Schmidt and Simone, 1996), and other existing models of collaboration (for example, Annett and Cunningham (2000), and Gutwin and Greenberg (2002)), the concepts of communication, coordination, cooperation and control were isolated as potentially helpful. Bardram's (1998) split of collaborative work into coordination, cooperation and coconstruction and the associated transformations between these levels (Figure 3) was identified as the most useful way to view collaboration to complement the model of communication. It crystallizes what has been identified as important by various other approaches to studying collaborative work, and places them in a helpful structure. The breakdown of collaboration into levels with movements between them provides a dynamic view of the collaborative process. These levels clearly capture different aspects of collaboration that require different relationships between the participants involved in the work to succeed. Thus, this structure provides a useful context for the model of communication because it identifies what shapes the requirements of what this model shows to enable successful collaboration. The levels can also be easily described in terms of goals, planning and actions. This helps to clarify what is involved in work and what is required in the extended model of communication, which already uses these terms to describe cognition.

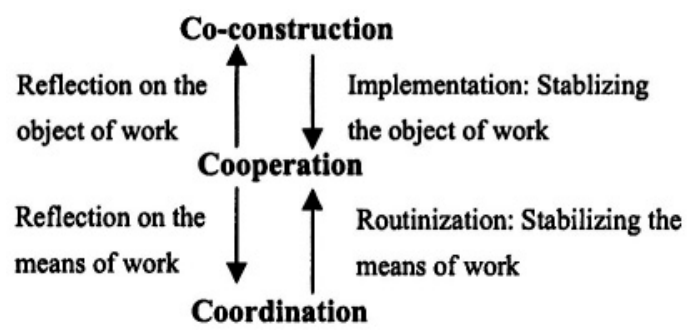

Figure 3. The Dynamics of Collaborative Work (from Bardram, 1998)

In Coordinated work, goals and plans already exist and participants focus on the performance of their individual actions. In Cooperative work the participants' focus is on their common goal and how to achieve it rather than their individual roles and actions. This level of work is more concerned with the planning of work because the way to achieve the common goal may either not exist or be open to change. Cooperation is therefore concerned with the participants' ability to work with each other to find a way to achieve their shared goal. Finally, Co-construction is about the goals of work because it involves participants coming together to question the whole aim 
of the work and hence goals and sub-goals associated with this. All collaborative work involves all three levels. Therefore all three must be considered to analyse collaboration thoroughly for possible error.

Movement between levels of work can also be explained with reference to group goals, plans and actions. At the coordination level goals and plans are set. If there is a problem with performing the prescribed actions, individuals may have to move into the cooperation level to plan together to find a solution. If the goals of the activities are brought into question then the participants move to the co-construction level of work. Movement down the levels is caused by resolving the problems with the goals and constructing plans to perform the work.

Now that a useful structure for describing collaborative work has been identified, the model of communication can be analysed in the context of each of these three different levels of work (with their goals, plans and actions mapping) to create a systematic error identification framework. This is created by isolating what is important within the model of collaboration according to the requirements of each of the levels (or aspects) of collaboration. The failure of collaboration is related to the failure of these requirements.

\section{NEXT STEPS TOWARDS A NEW ERROR IDENTIFICATION FRAMEWORK}

A systematic means of examining what is important within the model of collaboration is needed, according to the requirements of each aspect of collaboration (coordination, cooperation and co-construction). For all aspects of collaborative work there are certain relationships between the elements involved in the work that are important for success. The term 'collaborative error' describes errors that occur because of problems relating to the ability of individuals to perform activities with one another as a group to fulfil group aims. Therefore, the key relationships to examine are concerned with how the individuals relate to one another and the group as a whole.

For collaboration to work effectively it is necessary to consider the relationships between the individuals involved. Individuals must often have shared or complementary goals, interpret a situation in the same way, or perform actions that complement each other. Disagreement between individuals is a potential indication or cause of error. It is also necessary to consider how individuals relate to the group as a whole. Error could be caused by a problem with an individual's understanding of the group's goals or how they are to be reached. Failure could also occur because of the failure 
of an individual to comply with the group's aims. The group must also be considered as a whole. The following key relationships in the success of collaborative work have been identified:

- Individual $\leftrightarrow$ Individual (how participants relate to each other)

- Individual $\leftrightarrow$ Group (how participants relate to the group as a whole)

- Group (participants' collective actions have to achieve group purposes)

These relationships are built through, and rely on the communication shown in the model. Hence, they are a useful structure with which to explain the requirements for each level (or aspect) of collaboration, in terms of the model used. All of these relationships are important for each of the three aspects of collaboration (coordination, cooperation and co-construction) for different reasons. Table 2 shows how these relationships need to be considered to examine collaboration.

Table 2. The Structure of the Framework to Analyse the Model of Collaboration

\begin{tabular}{|c|c|c|c|c|c|c|c|c|}
\hline \multicolumn{9}{|c|}{ Collaboration } \\
\hline \multicolumn{3}{|c|}{ Coordination } & \multicolumn{3}{|c|}{ Cooperation } & \multicolumn{3}{c|}{ Co-construction } \\
\hline $\mathrm{I} \leftrightarrow \mathrm{I}$ & $\mathrm{I} \leftrightarrow$ Group & Group & $\mathrm{I} \leftrightarrow \mathrm{I}$ & $\mathrm{I} \leftrightarrow$ Group & Group & $\mathrm{I} \leftrightarrow \mathrm{I}$ & $\mathrm{I} \leftrightarrow$ Group & Group \\
\hline
\end{tabular}

The requirements of the relationships between individuals and the group can be expressed more precisely in terms of cognitive stages. For example:

- Interpretation $(I) \leftrightarrow$ Interpretation $(I)$ (e.g. individuals in the group may have to have the same interpretation of something)

- Plans $(I) \leftrightarrow$ Goals(Group) (e.g. individuals plans may have to contribute to or be in agreement with the group goals)

The relationships between individuals and the group can be examined more systematically and in detail this way. To identify specific ways in which collaboration may fail, the cognitive stages in the model are first used to establish precisely what is required of the relationships between individuals and the group for each aspect of collaboration (coordination, cooperation and co-construction). So to identify the requirements of collaboration using these cognitive stage relationships, the requirements of these relationships for each aspect of collaboration are considered, i.e. the requirements of $I \leftrightarrow I, I \leftrightarrow$ Group, and Group in coordination are described in terms of cognitive stage relationship requirements, the requirements of cooperation are described in terms of cognitive stage relationship requirements, and the requirements for co-construction are described in terms of cognitive stage relationships. For example:

- Coordination:

- $\operatorname{actions}(I) \leftrightarrow$ actions $(I)$ etc.

- goals(I)↔goals(Group) etc.

- actions (Group) etc. 
For the purposes of this analysis, the group is considered to have goals, plans and actions, and individuals have the whole of Norman's cognitive loop (2002) as shown in the model of collaboration (Figure 2).

Exactly what is required of which cognitive stage relationships depends on the aspect of work being examined. The coordination, cooperation, coconstruction split provides the context of the wider activity with which to understand how the individuals and group have to relate to each other. What is important about these cognitive stage relationships, and in what way, will be different for different aspects of work because of the different structures of the work. Each aspect of collaboration has a different level of completeness of group goals and plans, and different 'awareness of each other' requirements. What is required of a cognitive stage relationship for each aspect of work to be successful can be established based on the description of each aspect in terms of goals, plans and actions. For example, the goals $(I) \leftrightarrow$ goals $(I)$ relationship for cooperation requires that a goal be shared, whereas for coordination it is only necessary that the goals are complementary so that they do not conflict. In the context of Table 2 , the cognitive stage relationships can therefore be used to consider issues such as: the degree of awareness required of each others' goals and activities, the potential need for shared interpretation or development of shared understanding, the degree of awareness required of group goals and plans, and what controls the coordination of individual work to fulfil the whole group aims. The systematic examination of what is important about these relationships for each aspect of work and why it is important will help to clarify the requirements of collaborative work to function effectively and therefore also ways in which it may fail. Potential errors of collaboration are framed as breakdowns of these cognitive stage relationships. The need to move between these levels of work will also be considered, as they may be a source of error.

Failure guidewords can be applied to these relationships to establish more specific types of failure. For example:

- Cooperation:

- goals $(I) \leftrightarrow$ goals $(I)$ - conflict (individuals goals conflict)

- actions(I)↔plans(Group) - Incorrect (one or more individuals are performing actions that disagree with the group plans)

- goals(Group) - Failure (the goals of individuals in the group do not come together to fulfil the group goals)

The failure guidewords appropriate to the cognitive stage relationships for each aspect of work depend on the requirements of these relationships for each aspect. Potential failures can be identified according to how the requirements of cognitive stage relationships may fail within each aspect of 
work. This process produces a list of possible ways in which collaboration may fail.

Having identified which cognitive stage relationships are important for each aspect of work and how they are important, and then having considered how these may fail, it is necessary to examine the possible reasons why these may fail within each aspect of collaboration. This will allow the creation of error analysis questions. Deeper examination of the enhanced model of communication, using the framework in Table 2, allows possible causes of failure to be considered systematically. This involves taking the requirements of each aspect of work for success (in terms of cognitive stage relationships as discussed above) and then identifying the elements responsible for maintaining these cognitive stage relationships in that aspect of work (for example, the degree of awareness of certain aspects of work required to create shared understanding, or rules for work). This information will originate from different places in different forms of work. What the participants need to create these cognitive stage relationships successfully (for example, instructions or rules and environmental triggers for these in coordination, or a shared awareness of past, present, and future issues for cooperation) can be used to question design issues. The model of communication allows the systematic consideration of how these requirements may be supported, or of design reasons why they may fail. Error analysis questions can then be constructed that tackle these design issues raised using the model.

\section{CONCLUSION AND FUTURE WORK}

An iterative approach to designing an error analysis method for collaborative work has been taken. From an evaluation of the original approach to using a model of collaboration to create error analysis questions it was shown that the process of creating the CHLOE error analysis led to questions that did not tackle collaborative failure in enough depth. The results of the evaluation have been used to guide a new approach to creating error analysis questions. Through enhancing the model of communication, identifying a breakdown of collaboration to provide context for this model, and isolating key relationships in collaboration, an improved analysis framework has been created that can structure the description of the requirements of collaborative work. This framework will now be used as a more systematic and detailed way of understanding collaborative work and identifying possible failures within it, upon which to build an improved error analysis method. 


\section{ACKNOWLEDGEMENTS}

Angela Miguel is supported by an EPSRC studentship on the DIRC project (http://www.dirc.org.uk), UK EPSRC Grant N13999. We would also like to thank our anonymous referees for their insightful comments.

\section{REFERENCES}

Annett J. \& Cunningham D. (2000) Analysing Command Team Skills In Cognitive Task Analysis, Shraagen J. M., Chipman S. F., Shalin V. L. Eds. LEA.

Bardram, Jakob E. (1998): Designing for the Dynamics of Cooperative Work Activities. In Proceedings of 1998 ACM Conference on Computer Supported Cooperative Work, Seattle, Washington, USA. ACM Press.

Chudleigh, M.F, \& Clare, J.N. (1993) The Benefits of SUSI: Safety Analysis of User System Interaction. In J. Gorski (Ed.) SAFECOMP'93. Proceedings of the $12^{\text {th }}$ International Conference on Computer Safety, Reliability \& Security, Poznan-Kiekrz, Poland. Springer Verlag.pp 123-132.

Clark, H. H. (1996) Using Language. Cambridge, UK. Cambridge University Press.

Dix, A., Finlay, J., Abowd, G. \& Beale, R. (1998) Human-Computer Interaction $2^{\text {nd }}$ Ed. Prentice Hall Europe, Harlow, Essex.

Gutwin, C. \& Greenberg, S. (2002) A Descriptive Framework of Workspace Awareness for Real-Time Groupware Computer Supported Cooperative Work 11,411-446.

Hollnagel, E. (1998) Cognitive Reliability and Error Analysis Method (CREAM). Elsevier Science Ltd.

Hutchins, E. (1995) Cognition in the Wild. MIT Press.

Kletz, A. (1999) Hazop and Hazan: Identifying and Assessing Process Industry Hazards.4 $4^{\text {th }}$ Ed. Rugby, UK

Laurillau, Y. \& Nigay, L. (2002) Clover Architecture for Groupware. In Proceedings of 2002 ACM Conference on Computer Supported Cooperative Work, New Orleans, Louisianna, USA. ACM Press. pp.236-245.

Malone, T. W. \& Crowston, K. (1990) What is Coordination Theory and How Can it Help Design Cooperative Work Systems. Proc. of the $3^{\text {rd }}$ Conf. on CSCW, ACM, p 357-370.

Miguel, A. \& Wright, P. (2003) CHLOE: A Technique for Analysing Collaborative Systems. Proc. of the 9th CSAPC, G. van der Veer \& J. F. Hoorn (Eds), pp53-60.

Nardi, B. A. (1996) Context and Consciousness: Activity Theory and Human-Computer Interaction. MIT Press.

Norman, D. A. (2002) The Design of Everyday Things. Doubleday, New York.

Pocock, S., Harrison, M.D., Wright, P.C. and Johnson, P.D. (2001). THEA: a technique for human error assessment early in design. In M, Hirose (Ed.) IFIP TC 13 International Conference on Human-Computer Interaction. IOS Press. Ohmsha. pp. 247-254.

Schmidt K. \& Simone C. (1996) Coordination Mechanisms: Towards a Conceptual

Foundation of CSCW Systems Design, Computer Supported Cooperative Work (CSCW), vol. 5 , no. $2 / 3,1996$.

Segal L. D. (1995) Designing Team Workstations: The Choreography of Teamwork In Local Applications of the Ecological Approach to Human-Machine Systems Vol. 2, Hancock P., Flach J., Caird J. \& Vicente K. Eds. LEA. 